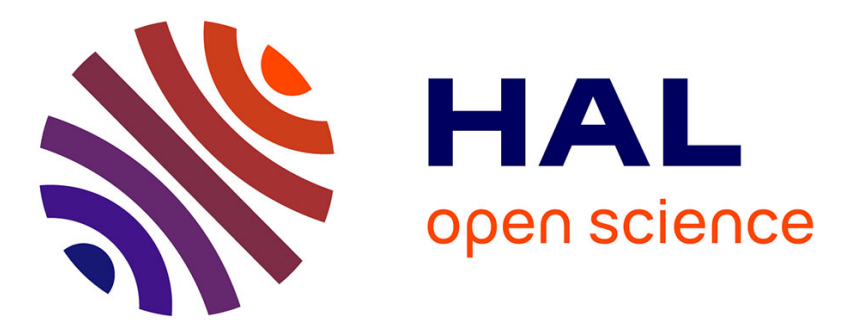

\title{
Derivation of the theoretical performance of a Tensor MUSIC algorithm
}

\author{
Philippe Forster, Guillaume Ginolhac, Maxime Boizard
}

\section{To cite this version:}

Philippe Forster, Guillaume Ginolhac, Maxime Boizard. Derivation of the theoretical performance of a Tensor MUSIC algorithm. Signal Processing, 2016, 129 (12), pp.97-105. 10.1016/j.sigpro.2016.05.033 . hal-01391933

\section{HAL Id: hal-01391933 https://hal.science/hal-01391933}

Submitted on 4 Nov 2016

HAL is a multi-disciplinary open access archive for the deposit and dissemination of scientific research documents, whether they are published or not. The documents may come from teaching and research institutions in France or abroad, or from public or private research centers.
L'archive ouverte pluridisciplinaire HAL, est destinée au dépôt et à la diffusion de documents scientifiques de niveau recherche, publiés ou non, émanant des établissements d'enseignement et de recherche français ou étrangers, des laboratoires publics ou privés. 


\title{
Derivation of the theoretical performance of a Tensor MUSIC Algorithm
}

\author{
P. Forster, G. Ginolhac, M. Boizard ${ }^{1}$ \\ SATIE, ENS Cachan, CNRS, UniverSud, 61, av President Wilson, F-94230 Cachan, France \\ LISTIC, Université de Savoie Mont-Blanc, B.P. 8043974944 Annecy le Vieux Cedex, France
}

\begin{abstract}
In this paper, we derive the theoretical performance of a Tensor MUSIC algorithm based on the Higher Order Singular Value Decomposition (HOSVD), that was previously developed to estimate the Direction Of Arrival (DOA) and the polarization parameters of polarized sources. The derivation of this result is done via a perturbation analysis and allows to obtain the theoretical Mean Square Error (MSE) on the DOA and the polarization parameters. The proposed result is also shown to be valid for the Long Vector MUSIC algorithm, i.e. when the multidimensional samples are unfolded into a long vector. The agreement between theoretical and empirical MSEs is illustrated through Monte Carlo simulations.
\end{abstract}

Keywords: Tensor MUSIC, HOSVD, DOA, polarization sources, Theoretical Mean Square Error, Perturbation Analysis

\section{Introduction}

An increasing number of signal processing applications deal with multidimensional data. One can cite for example array processing [1], channel egalisation [2], polarimetric STAP [3], polarized seismic sources localization [4], multidimensional harmonic retrieval [5][6] or MIMO coding [7]. Multilinear algebra [8][9][10][11] provides an appropriate framework to exploit these data since it preserves the multidimensional information structure. Nevertheless, generalizing matrix-based algorithms to the multilinear algebra framework is not a trivial

\footnotetext{
Email address:

pforster@u-paris10.fr; guillaume.ginolhacduniv-smb. fr (P. Forster, G. Ginolhac, M. Boizard)
} 
task. In particular, there is no multilinear counterpart of the Singular Value Decomposition (SVD) that shares exactly the same properties. Notably, two main decompositions exist: CANDECOMP/PARAFAC (CP) [12] which allows to define a rank like for SVD (and which is especially used for its remarkable identifiability property under some conditions) and the Higher Order Singular Value Decomposition (HOSVD) [8], derived from the Tucker decomposition [13], which keeps the orthogonality properties of the SVD.

This paper focuses on the problem of polarized source parameters estimation (Direction Of Arrival (DOA) and polarization parameters) by using the wellknown MUltiple SIgnal Classification (MUSIC) algorithm [14] for multidimensional configurations. Classically the received data correspond to a vector whose dimension is the number of sensors. If an additional dimension is available, e.g. polarization, Doppler frequency, or multiple emitters/sensors for a MIMO configuration, the data turn out to be multidimensional. In this case, it remains possible, as proposed in [4] to apply the classical MUSIC algorithm by unfolding the data into vectors. This approach will be denoted Long Vector MUSIC (LV-MUSIC) in this paper. However this algorithm does not fully exploit the multidimensional structure of the covariance tensor of the observations. Moreover, since this approach increases the size of the data, the number of samples needed to reach acceptable performances is increased likewise (typically around twice the size of the unfolded vector to accurately estimate the covariance matrix [15]). Therefore, the development of new estimation algorithms, accounting for multidimensionally structured data, is needed. In this framework, several possibilities, depending on the considered tensor decomposition, have been explored.

Initially, several approaches based on the $\mathrm{CP}$ decomposition were proposed, as in [16] where the localization system is composed of multiple electromagnetic sensors. In [17], the authors have considered the same model with correlated sources and have shown that the joint use of space and polarimetric smoothing solves the correlation issue. Noteworthy, treatments based on CP decomposition always require to study the identifiability properties of the problem, in order to ensure the unicity of the solution.

In a former work [18], we have developed a Tensor MUSIC algorithm based on the HOSVD, denoted T-MUSIC. This algorithm is adapted from [6], which considers a multidimensional Vandermonde-type decomposition in order to perform multidimensional harmonic retrieval. The T-MUSIC algorithm is based on the best reduced rank approximation of a higher order tensor [19]. Recently, the T-MUSIC algorithm has been used for nested vector-sensor array [20]. In this paper, we consider the electromagnetic model of [4][18]. 
As for the vector case, an important issue is to determine the performance of newly proposed tensor algorithms. In order to avoid relying on extensive MonteCarlo simulations prior to system designing, one ideally wants access to the theoretical performance, with closed-form expressions. Therefore, we propose in this paper to derive the theoretical performance of the T-MUSIC algorithm developed in [18]. We will additionally show that the obtained result encompasses the LV-MUSIC [4] approach. Several recent works, relying on singular vector perturbation [21], have studied the performance of Tensor ESPRIT approaches in the domain of multi-dimensional harmonic retrieval [22][23]. In this paper, our derivation is based on the perturbation method proposed in [24], already used in the vector case to determine the theoretical signal to noise ratio loss of adaptive low rank filters in [25][26].

This paper is organized as follows. Section II presents the multilinear algebra tools used in the paper. Then, the data model and the associated T-MUSIC algorithms are described in section III. The main contribution about theoretical performance is contained in section IV. In section V, the proposed theoretical result is validated through Monte-Carlo simulations. Section VI draws some conclusions of this study.

The following convention is adopted: scalars are denoted by italic letters, vectors by lower-case bold-face letters, matrices by bold-face capitals, and tensors are written by bold-face calligraphic letters. We use the superscripts ${ }^{T}$, for the transpose operator, ${ }^{H}$, for Hermitian transposition, ${ }^{*}$ for complex conjugation and $\|$.$\| , the euclidean norm. The mathematical expectation is denoted by E[$.$] and$ the Kronecker product is denoted by $\otimes \cdot \mathcal{C N}(\mathbf{a}, \mathbf{R})$ is a complex Gaussian vector of mean a and of covariance matrix $\mathbf{R} . \mathcal{N}_{c}\left(a, \sigma^{2}\right)$ is complex Gaussian random variable of mean $a$ and variance $\sigma^{2}$.

\section{Some multilinear algebra tools}

This section contains the main multilinear algebra tools used in this paper. Let $\mathcal{A}, \mathcal{B} \in \mathbb{C}^{I_{1} \times I_{2} \times I_{3} \times I_{4}}$, be two 4 -dimensional tensors and let $a_{i_{1} i_{2} i_{3} i_{4}}, b_{i_{1} i_{2} i_{3} i_{4}}$ be their elements. The following operators are used for this paper. For more details, especially the case of $n$-order tensors, we refer the reader to $[10,8,11]$.

\subsection{Unfolding}

In this paper, we consider the following three unfoldings:

- vector: vec transforms a tensor $\mathcal{A}$ into a vector, $\operatorname{vec}(\mathcal{A}) \in \mathbb{C}^{I_{1} I_{2} I_{3} I_{4}}$. We denote $v e c^{-1}$, the inverse vector operator. 
- matrix: Let us denote $[\mathcal{A}]_{n}, n=1, \ldots, 4$, the operator which transforms the tensor $\mathcal{A}$ into a matrix by concatenating the different slices of the tensor along the nth mode. For example, $[\mathcal{A}]_{1} \in \mathbb{C}^{I_{1} \times I_{2} I_{3} I_{4}}$. The inverse operator always exists if the way the tensor has been unfolded is known.

- square matrix: this operator transforms the square tensor $\mathcal{R} \in \mathbb{C}^{I_{1} \times I_{2} \times I_{1} \times I_{2}}$ into a square matrix, $\operatorname{SqMat}(\mathcal{R}) \in \mathbb{C}^{I_{1} I_{2} \times I_{1} I_{2}}$. SqMat ${ }^{-1}$ denotes the inverse square matrix operator.

\subsection{Products}

- Scalar product of two tensors:

$<\mathcal{A}, \mathcal{B}>=\sum_{i_{1}} \sum_{i_{2}} \sum_{i_{3}} \sum_{i_{4}} b_{i_{1} i_{2} i_{3} i_{4}}^{*} a_{i_{1} i_{2} i_{3} i_{4}}$.

- From the scalar product, we define the Frobenius-norm: $\|\mathcal{A}\|=\sqrt{\langle\mathcal{A}, \mathcal{A}\rangle}$

- The $n$-mode product ( $n=1, \ldots, 4$ ) consists of multiplying an $I_{1} \times I_{2} \times$ $I_{3} \times I_{4}$-tensor $\mathcal{A}$ by an $J_{n} \times I_{n}$ matrix $\mathbf{E}\left(I_{n}\right.$ could be equal to $I_{1}, I_{2}, I_{3}$ or $\left.I_{4}\right):$

$\left(\mathcal{A} \times{ }_{n} \mathbf{E}\right)_{i_{1} j_{n} i_{3} i_{4}}=\sum_{i_{n}} a_{i_{1} i_{2} i_{3} i_{4}} e_{j_{n} i_{n}}$. The previous equation is given for $n=2$.

- Outer product of two tensors: $\mathcal{E}=\mathcal{A} \circ \mathcal{B}$

$\in \mathbb{C}^{I_{1} \times I_{2} \times I_{3} \times I_{4} \times I_{1} \times I_{2} \times I_{3} \times I_{4}}$ with $e_{i_{1} i_{2} i_{3} i_{4} j_{1} j_{2} j_{3} j_{4}}=a_{i_{1} i_{2} i_{3} i_{4}} \cdot b_{j_{1} j_{2} j_{3} j_{4}}$. For example, for two vectors, $\mathbf{a}, \mathbf{b}$, their outer product $\mathbf{a} \circ \mathbf{b}$ is a rank-1 matrix.

\subsection{Higher Order Singular Value Decomposition}

The Higher Order Singular Value Decomposition (HOSVD) decomposes a 4order tensor $\mathcal{A}$ as follows

$$
\mathcal{A}=\mathcal{K} \times{ }_{1} \mathbf{U}^{(1)} \times{ }_{2} \mathbf{U}^{(2)} \times_{3} \mathbf{U}^{(3)} \times_{4} \mathbf{U}^{(4)}
$$

where $\forall n=1 \ldots 4, \mathbf{U}^{(n)} \in \mathbb{C}^{I_{n} \times I_{n}}$ is an orthonormal matrix and where $\mathcal{K} \in$ $\mathbb{C}^{I_{1} \times I_{2} \times I_{3} \times I_{4}}$ is the core tensor, which satisfies the all-orthogonality conditions [8]. The matrix $\mathbf{U}^{(n)}$ is given by the SVD of the $n$-dimension unfolding tensor, $[\mathcal{A}]_{n}=$ $\mathbf{U}^{(n)} \boldsymbol{\Sigma}^{(n)} \mathbf{V}^{(n) H}$.

Furthermore, if $\mathcal{A}$ is an Hermitian tensor, i.e. $I_{1}=I_{3}, I_{2}=I_{4}$ and $a_{i_{1}, i_{2}, i_{3}, i_{4}}=$ $a_{i_{3}, i_{4}, i_{1}, i_{2}}^{*}, \forall i_{1}, i_{2}, i_{3}, i_{4}$, the HOSVD of $\mathcal{A}$ is written [5]:

$$
\mathcal{A}=\mathcal{K} \times{ }_{1} \mathbf{U}^{(1)} \times_{2} \mathbf{U}^{(2)} \times{ }_{3} \mathbf{U}^{(1) *} \times_{4} \mathbf{U}^{(2) *} .
$$




\subsection{Covariance Tensor and Sample Covariance Tensor}

Definition. Let $\mathbf{X} \in \mathbb{C}^{I_{1} \times I_{2}}$ be a random matrix, the covariance tensor, $\mathcal{R} \in$ $\mathbb{C}^{I_{1} \times I_{2} \times I_{1} \times I_{2}}$ is defined as [4]:

$$
\mathcal{R}=E\left[\mathbf{X} \circ \mathbf{X}^{H}\right]
$$

If we denote $\mathbf{x}=\operatorname{vec}(\mathbf{X})$, then the covariance matrix $\mathbf{R} \in \mathbb{C}^{I_{1} I_{2} \times I_{1} I_{2}}$ of $\mathbf{x}$ is linked to the covariance tensor by the following relation:

$$
\mathbf{R}=\operatorname{SqMat}(\mathcal{R}) .
$$

In many applications, the covariance tensor is unknown and has to be estimated from so-called secondary data, that are assumed to be independent and identically distributed. Let $\mathbf{X}(n) \in \mathbb{C}^{I_{1} \times I_{2}}$ be $N_{s}$ observations of $\mathbf{X}$. By analogy with the Sample Covariance Matrix (SCM), $\hat{\mathcal{R}} \in \mathbb{C}^{I_{1} \times I_{2} \times I_{1} \times I_{2}}$, the Sample Covariance Tensor (SCT) is defined as [5]:

$$
\hat{\mathcal{R}}=\frac{1}{N_{s}} \sum_{n=1}^{N_{s}} \mathbf{X}(n) \circ \mathbf{X}(n)^{H}
$$

\section{MUSIC Algorithms for polarized source}

\subsection{Polarized source model}

Let us consider an uniform linear array of $M$ sensors which can receive $N_{c}$ polarimetric channels and a polarized source impinging on the array. The onedimensional DOA of the source is denoted by $\theta$. The signal propagation along the spatial dimension of a polarimetric channel is modeled as follows:

$$
\mathbf{d}(\theta)=\left(1, e^{-2 i \pi \frac{d}{\lambda} \sin (\theta)}, \ldots, e^{-2 i \pi(M-1) \frac{d}{\lambda} \sin (\theta)}\right)^{T} \in \mathbb{C}^{M},
$$

where $d$ is the distance between two sensors and $\lambda$ the wavelength. On one sensor, we model the signal received in channel $n_{c}$ by multiplying its amplitude by $\rho_{c}$ and shifting its phase by $\varphi_{c}$ relative to the first channel (channel 0 ) as in many polarimetry applications ${ }^{1}$. Thus, for a single sensor, the signal behaviour along the polarization dimension can be modeled as [4]:

$$
\mathbf{p}(\boldsymbol{\rho}, \boldsymbol{\varphi})=\left(1, \rho_{1} e^{i \varphi_{1}}, \ldots, \rho_{N_{c}-1} e^{i \varphi_{N_{c}-1}}\right)^{T} \in \mathbb{C}^{N_{c}},
$$

\footnotetext{
${ }^{1}$ For example in polarimetric RADAR, 2 different polarized signals are emitted in $\mathrm{HH}$ and VV. These signals are received in 4 polarizations: $\mathrm{HH}, \mathrm{VV}, \mathrm{HV}, \mathrm{VH}$.
} 
where $\boldsymbol{\rho}=\left(\rho_{1}, \ldots, \rho_{N_{c}-1}\right)$ and $\boldsymbol{\varphi}=\left(\varphi_{1}, \ldots, \varphi_{N_{c}-1}\right)$ are the gain and the phase shift between channels. Combining equations (6) and (7), we define the steering vector $\mathbf{a}(\theta, \boldsymbol{\rho}, \boldsymbol{\varphi}) \in \mathbb{C}^{M N_{c}}$ modeling the signal propagation along the whole array:

$$
\mathbf{a}(\theta, \boldsymbol{\rho}, \boldsymbol{\varphi})=\mathbf{d}(\theta) \otimes \mathbf{p}(\boldsymbol{\rho}, \boldsymbol{\varphi}) .
$$

This steering vector is used in this given form for the vector approach. However, equations (6) and (7) can also be combined in order to obtain a steering matrix, $\mathbf{A}(\theta, \boldsymbol{\rho}, \boldsymbol{\varphi}) \in \mathbb{C}^{M \times N_{c}}$, defined as

$$
\mathbf{A}(\theta, \boldsymbol{\rho}, \boldsymbol{\varphi})=\mathbf{d}(\theta) \circ \mathbf{p}(\boldsymbol{\rho}, \boldsymbol{\varphi})=\mathbf{d}(\theta) \mathbf{p}^{T}(\boldsymbol{\rho}, \boldsymbol{\varphi}) .
$$

This model keeps the two-dimensional structure of the source and will be used to derive the proposed tensor MUSIC algorithm.

\subsection{Long Vector MUSIC}

Let us now consider $P$ independent zero-mean Gaussian sources with unit variance. We assume that $N_{s}$ snapshots of the sources impinging the array are available. The $n$-th snapshot, denoted $\mathbf{x}(n) \in \mathbb{C}^{M N_{c}}$ is written

$$
\mathbf{x}(n)=\sum_{p=1}^{P} s_{p}(n) \mathbf{a}\left(\theta_{p}, \boldsymbol{\rho}_{p}, \boldsymbol{\varphi}_{p}\right)+\mathbf{n}(n)
$$

where $\mathbf{a}\left(\theta_{p}, \boldsymbol{\rho}_{p}, \boldsymbol{\varphi}_{p}\right)$ is the steering vector of the $p$-th source and $s_{p}(n) \sim \mathcal{N}_{c}\left(0, \sigma_{p}^{2}\right)$ the zero-mean complex Gaussian-distributed random amplitude of the $p$-th source. $\mathbf{n}(n) \sim \mathcal{C N}\left(\mathbf{0}, \sigma^{2} \mathbf{I}_{M N_{c}}\right)$ is a zero-mean complex white Gaussian noise vector.

Let $\mathbf{R}=E\left[\mathbf{x x}^{H}\right] \in \mathbb{C}^{M N_{c} \times M N_{c}}$, be the covariance matrix of the data. Since $\mathbf{R}$ is unknown in practice, it has to be estimated by the SCM, $\hat{\mathbf{R}}=\frac{1}{N_{s}} \sum_{n=1}^{N_{s}} \mathbf{x}(n) \mathbf{x}^{H}(n)$. The Long Vector MUSIC (LV-MUSIC) algorithm is performed as the classical MUSIC algorithm in 3 steps. First the SVD of $\hat{\mathbf{R}}$ is computed

$$
\hat{\mathbf{R}}=\hat{\mathbf{U}} \hat{\mathbf{\Sigma}} \hat{\mathbf{U}}^{H} \text {. }
$$

Then, $\hat{\mathbf{U}}$ is truncated into $\hat{\mathbf{U}}_{0}$, keeping the $\left(M N_{c}-P\right)$ last columns of $\hat{\mathbf{U}}$ corresponding to the $M N_{c}-P$ eigenvalues. Thus, the columns of $\hat{\mathbf{U}}_{0}$ represent an orthonormal basis of the estimated noise subspace. Finally, the parameters of the sources are obtained by minimizing the following criterion:

$$
\left\{\hat{\theta}_{p}^{L V-M U S I C}, \hat{\boldsymbol{\rho}}_{p}^{L V-M U S I C}, \hat{\boldsymbol{\varphi}}_{p}^{L V-M U S I C}\right\}=\arg \min _{(\theta, \boldsymbol{\rho}, \boldsymbol{\varphi})}\left(\hat{H}_{L V}(\theta, \boldsymbol{\rho}, \boldsymbol{\varphi})\right)
$$

where

$$
\begin{aligned}
\hat{H}_{L V}(\theta, \boldsymbol{\rho}, \boldsymbol{\varphi}) & =\left\|\hat{\mathbf{U}}_{0}^{H} \mathbf{a}(\theta, \boldsymbol{\rho}, \boldsymbol{\varphi})\right\|^{2} \\
& =\operatorname{tr}\left(\hat{\boldsymbol{\Pi}} \mathbf{a}(\theta, \boldsymbol{\rho}, \boldsymbol{\varphi}) \mathbf{a}(\theta, \boldsymbol{\rho}, \boldsymbol{\varphi})^{H}\right)
\end{aligned}
$$

where $\hat{\boldsymbol{\Pi}}=\hat{\mathbf{U}}_{0} \hat{\mathbf{U}}_{0}^{H}$. 


\subsection{Tensor MUSIC}

As in the previous subsection, let us now consider $P$ independent zero-mean Gaussian sources with unit variance and $N_{s}$ snapshots of the sources impinging the array. For the matrix observation model, the $n$-th snapshot, denoted $\mathbf{X}(n) \in$ $\mathbb{C}^{M \times N_{c}}$ is modeled by

$$
\mathbf{X}(n)=\sum_{p=1}^{P} s_{p}(n) \mathbf{A}\left(\theta_{p}, \boldsymbol{\rho}_{p}, \boldsymbol{\varphi}_{p}\right)+\mathbf{N}(n)
$$

where $\mathbf{A}\left(\theta_{p}, \boldsymbol{\rho}_{p}, \boldsymbol{\varphi}_{p}\right)$ is the steering matrix (9) of the $p$-th source, $s_{p}(n) \sim \mathcal{N}_{c}\left(0, \sigma_{p}^{2}\right)$ the zero-mean complex Gaussian-distributed random amplitude of the $p$-th source. $\mathbf{N}(n)$ is a complex Gaussian matrix and $\operatorname{vec}(\mathbf{N}(n)) \sim \mathcal{C N}\left(\mathbf{0}, \sigma^{2} \mathbf{I}_{M N_{c}}\right)$. Let $\mathcal{R}=E\left[\mathbf{X} \circ \mathbf{X}^{*}\right] \in \mathbb{C}^{M \times N_{c} \times M \times N_{c}}$ be the covariance tensor. Similarly with the vector approach $\mathcal{R}$ is unknown in practice, it has to be estimated by the SCT, $\hat{\mathcal{R}}=\frac{1}{N_{s}} \sum_{n=1}^{N_{s}} \mathbf{X}(n) \circ \mathbf{X}(n)^{*}$.

By analogy with the vector case, the Tensor MUSIC (T-MUSIC) algorithm is derived as follows. First $\hat{\mathcal{R}}$ is decomposed using the HOSVD procedure as:

$$
\hat{\mathcal{R}}=\hat{\mathcal{K}} \times{ }_{1} \hat{\mathbf{U}}^{(1)} \times{ }_{2} \hat{\mathbf{U}}^{(2)} \times{ }_{3} \hat{\mathbf{U}}^{(1) *} \times_{4} \hat{\mathbf{U}}^{(2) *} .
$$

Then $\hat{\mathbf{U}}^{(1)}$ (respectively $\hat{\mathbf{U}}^{(2)}$ ) is truncated into $\hat{\mathbf{U}}_{0}^{(1)}$ (respectively $\hat{\mathbf{U}}_{0}^{(2)}$ ) by keeping the $(M-P)$ last columns of $\hat{\mathbf{U}}^{(1)}$ (respectively the $\left(N_{c}-P\right)$ last columns of $\left.\hat{\mathrm{U}}^{(2)}\right)$. It is well known that this solution for estimating the noise subspace is not optimal in the least squares sense. However, it is a correct approximation in most cases [5][19] and it is easy to implement, which is why the use of iterative algorithms will not be considered in this paper. Finally the sources parameters are obtained by minimizing the following criterion:

$$
\left\{\hat{\theta}_{p}^{T-M U S I C}, \hat{\boldsymbol{\rho}}_{p}^{T-M U S I C}, \hat{\boldsymbol{\varphi}}_{p}^{T-M U S I C}\right\}=\arg \min _{(\theta, \boldsymbol{\rho}, \boldsymbol{\varphi})}\left(\hat{H}_{T}(\theta, \boldsymbol{\rho}, \boldsymbol{\varphi})\right)
$$

where

$$
\hat{H}_{T}(\theta, \boldsymbol{\rho}, \boldsymbol{\varphi})=\left\|\mathbf{A}(\theta, \boldsymbol{\rho}, \boldsymbol{\varphi}) \times_{1} \hat{\mathbf{U}}_{0}^{(1) H} \times_{2} \hat{\mathbf{U}}_{0}^{(2) H}\right\|^{2} .
$$

This criterion can be re-expressed in a different form according to the following lemma.

Lemma 3.1. The criterion $\hat{H}_{T}(\theta, \boldsymbol{\rho}, \boldsymbol{\varphi})$ can be rewritten as follows:

$$
\hat{H}_{T}(\theta, \boldsymbol{\rho}, \boldsymbol{\varphi})=\operatorname{tr}\left(\hat{\boldsymbol{\Pi}}_{1} \mathbf{d}(\theta) \mathbf{d}^{H}(\theta)\right) \operatorname{tr}\left(\hat{\boldsymbol{\Pi}}_{2} \mathbf{p}(\boldsymbol{\rho}, \boldsymbol{\varphi}) \mathbf{p}^{H}(\boldsymbol{\rho}, \boldsymbol{\varphi})\right)
$$

where $\hat{\mathbf{\Pi}}_{1}=\hat{\mathbf{U}}_{0}^{(1)} \hat{\mathbf{U}}_{0}^{(1) H}$ and $\hat{\mathbf{\Pi}}_{2}=\hat{\mathbf{U}}_{0}^{(2)} \hat{\mathbf{U}}_{0}^{(2) H}$. The criterion is therefore separable and estimation of DOAs and polarization parameters can be performed independently. 
PROOF.

$$
\begin{aligned}
\hat{H}_{T}(\theta, \boldsymbol{\rho}, \boldsymbol{\varphi}) & =\left\|\mathbf{A}(\theta, \boldsymbol{\rho}, \boldsymbol{\varphi}) \times{ }_{1} \hat{\mathbf{U}}_{0}^{(1) H} \times{ }_{2} \hat{\mathbf{U}}_{0}^{(2) H}\right\|^{2} \\
& =\left\|\hat{\mathbf{U}}_{0}^{(1) H} \mathbf{A}(\theta, \boldsymbol{\rho}, \boldsymbol{\varphi}) \hat{\mathbf{U}}_{0}^{(2) *}\right\|^{2} \\
& =\operatorname{tr}\left(\hat{\mathbf{U}}_{0}^{(2) T} \mathbf{A}^{H}(\theta, \boldsymbol{\rho}, \boldsymbol{\varphi}) \hat{\mathbf{U}}_{0}^{(1)} \hat{\mathbf{U}}_{0}^{(1) H} \mathbf{A}(\theta, \boldsymbol{\rho}, \boldsymbol{\varphi}) \hat{\mathbf{U}}_{0}^{(2) *}\right) \\
& =\operatorname{tr}\left(\hat{\mathbf{U}}_{0}^{(2) T} \mathbf{A}^{H}(\theta, \boldsymbol{\rho}, \boldsymbol{\varphi}) \hat{\boldsymbol{\Pi}}_{1} \mathbf{A}(\theta, \boldsymbol{\rho}, \boldsymbol{\varphi}) \hat{\mathbf{U}}_{0}^{(2) *}\right) \\
& =\operatorname{tr}\left(\hat{\boldsymbol{\Pi}}_{1} \mathbf{A}(\theta, \boldsymbol{\rho}, \boldsymbol{\varphi}) \hat{\mathbf{U}}_{0}^{(2) *} \hat{\mathbf{U}}_{0}^{(2) T} \mathbf{A}^{H}(\theta, \boldsymbol{\rho}, \boldsymbol{\varphi})\right) \\
& =\operatorname{tr}\left(\hat{\boldsymbol{\Pi}}_{1} \mathbf{A}(\theta, \boldsymbol{\rho}, \boldsymbol{\varphi}) \hat{\boldsymbol{\Pi}}_{2}^{T} \mathbf{A}^{H}(\theta, \boldsymbol{\rho}, \boldsymbol{\varphi})\right) \\
& =\operatorname{tr}\left(\hat{\boldsymbol{\Pi}}_{1} \mathbf{d}(\theta) \mathbf{p}^{T}(\boldsymbol{\rho}, \boldsymbol{\varphi}) \hat{\boldsymbol{\Pi}}_{2}^{T} \mathbf{p}^{*}(\boldsymbol{\rho}, \boldsymbol{\varphi}) \mathbf{d}^{H}(\theta)\right) \\
& =\operatorname{tr}\left(\left(\mathbf{d}^{H}(\theta) \hat{\boldsymbol{\Pi}} \mathbf{d}(\theta)\right)\left(\mathbf{p}^{T}(\boldsymbol{\rho}, \boldsymbol{\varphi}) \hat{\boldsymbol{\Pi}}_{2}^{T} \mathbf{p}^{*}(\boldsymbol{\rho}, \boldsymbol{\varphi})\right)\right) \\
& =\operatorname{tr}\left(\hat{\boldsymbol{\Pi}}_{1} \mathbf{d}^{H}(\theta) \mathbf{d}(\theta)\right) \operatorname{tr}\left(\hat{\boldsymbol{\Pi}}_{2}^{T} \mathbf{p}^{*}(\boldsymbol{\rho}, \boldsymbol{\varphi}) \mathbf{p}^{T}(\boldsymbol{\rho}, \boldsymbol{\varphi})\right) \\
& =\operatorname{tr}\left(\hat{\boldsymbol{\Pi}}_{1} \mathbf{d}(\theta) \mathbf{d}^{H}(\theta)\right) \operatorname{tr}\left(\hat{\boldsymbol{\Pi}}_{2} \mathbf{p}(\boldsymbol{\rho}, \boldsymbol{\varphi}) \mathbf{p}^{H}(\boldsymbol{\rho}, \boldsymbol{\varphi})\right)
\end{aligned}
$$

\section{Theoretical performance of LV-MUSIC and T-MUSIC}

This section is dedicated to the derivation of the theoretical performance of LV-MUSIC and T-MUSIC. These derivations are based on a perturbation analysis as the one proposed in [24] for the theoretical performance analysis of the classical vector MUSIC algorithm.

First, notice in Eqs (13) and (18) that both LV-MUSIC and T-MUSIC involve terms of the form

$$
\hat{h}(\boldsymbol{\mu})=\operatorname{tr}(\hat{\mathbf{\Pi}} \mathbf{F}(\boldsymbol{\mu}))
$$

where $\boldsymbol{\mu}=\left[\mu_{1}, \ldots, \mu_{J}\right]^{T} \in \mathbb{R}^{J}$ is an appropriate unknown parameter vector and matrix $\mathbf{F}$ is either equal to $\mathbf{a} \mathbf{a}^{H}, \mathbf{d d}^{H}$ or $\mathbf{p} \mathbf{p}^{H}$ (d, $\mathbf{p}$ and a are defined in (6), (7) and (8)).

For T-MUSIC, the criterion is the product of two different criteria $\hat{h}_{1}\left(\boldsymbol{\mu}_{\mathbf{1}}\right)$ and $\hat{h}_{2}\left(\boldsymbol{\mu}_{\mathbf{2}}\right)$ which follow from Lemma 3.1. For the $P$ sources, the estimated parameter vectors $\hat{\boldsymbol{\mu}}_{p}, p \in\{1, \ldots, P\}$ satisfy

$$
\hat{\boldsymbol{\mu}}_{p}=\arg \min _{\boldsymbol{\mu}_{p}} \hat{h}\left(\boldsymbol{\mu}_{p}\right)
$$

When projector onto the noise subspace $\Pi$ is substituted for $\hat{\Pi}$ in (19), the resulting criterion $h$ is minimized for the exact values of the parameter vector.

In the following subsection, we derive the theoretical Mean Square Error (MSE) of $\hat{\boldsymbol{\mu}}_{p}$ (estimation of $\boldsymbol{\mu}_{p}$ ) for this general form and the following subsections are 
devoted to the particular theoretical MSEs of the DOA and the polarization parameters $\rho$ and $\phi$ for both LV-MUSIC and T-MUSIC.

\subsection{General Result}

Let $L$ be the common dimension of $\boldsymbol{\Pi}, \mathbf{F}(\boldsymbol{\mu})$ and $\mathbf{R} \in \mathbb{C}^{L \times L}$. The estimation error on the covariance matrix or the covariance tensor acts as a perturbation, denoted by $\Delta \mathbf{R}$ and defined as $\Delta \mathbf{R}=\hat{\mathbf{R}}-\mathbf{R}$. This perturbation leads to another perturbation $\Delta \Pi$ defined by $\hat{\Pi}=\Pi-\Delta \Pi$. The resulting error on the estimated parameters is denoted by $\Delta \boldsymbol{\mu}_{p}=\hat{\boldsymbol{\mu}}_{p}-\boldsymbol{\mu}_{p}$ where $\boldsymbol{\mu}_{p}$ is the exact value of the parameter vector. We propose in this paper to compute the second-order statistics of $\Delta \boldsymbol{\mu}_{p}$. This derivation is decomposed into several propositions. Proposition 4.1 gives the link between the perturbations $\Delta \boldsymbol{\mu}_{p}$ and $\Delta \Pi$. Then, proposition 4.2 derives a closed form expression for the covariance matrix of $\Delta \boldsymbol{\mu}_{p}$ as a function of $\Delta \mathbf{R}$. Finally, the next subsection provides the final results by giving the expressions of the second-order statistics of $\Delta \mathbf{R}$ for both LV-MUSIC and T-MUSIC.

Proposition 4.1. Under the previous assumptions, the first order expression of $\Delta \boldsymbol{\mu}_{p}$ in terms of $\Delta \Pi$ is given by:

$$
\Delta \boldsymbol{\mu}_{p}=\mathrm{B}^{-1} \mathbf{g}
$$

where $\mathbf{g} \in \mathbb{C}^{J}$ and $\mathbf{B} \in \mathbb{C}^{J \times J}$ have elements $g(i)=\operatorname{tr}\left(\Delta \mathbf{\Pi} \frac{\partial \mathbf{F}}{\partial \mu_{i}}\left(\boldsymbol{\mu}_{p}\right)\right)$ and $B(i, j)=\operatorname{tr}\left(\boldsymbol{\Pi} \frac{\partial^{2} \mathbf{F}}{\partial \mu_{i} \partial \mu_{j}}\left(\boldsymbol{\mu}_{p}\right)\right)$.

PROOF. The vectors $\hat{\boldsymbol{\mu}}_{p}$ are the minima of the function $\hat{h}$, hence they satisfy:

$$
\nabla \hat{h}\left(\hat{\boldsymbol{\mu}}_{p}\right)=\mathbf{0}
$$

which means that for all $i=1 \ldots J$ :

$$
\operatorname{tr}\left(\hat{\boldsymbol{\Pi}} \frac{\partial \mathbf{F}}{\partial \mu_{i}}\left(\hat{\boldsymbol{\mu}}_{p}\right)\right)=0 .
$$

The above equation is satisfied by $\boldsymbol{\mu}_{p}$ when $\Pi$ is substituted for $\hat{\Pi}$ :

$$
\operatorname{tr}\left(\boldsymbol{\Pi} \frac{\partial \mathbf{F}}{\partial \mu_{i}}\left(\boldsymbol{\mu}_{p}\right)\right)=0
$$


The term $\frac{\partial \mathbf{F}}{\partial \mu_{i}}\left(\hat{\boldsymbol{\mu}}_{p}\right)$ in (23) can be approximated up to the first order with respect to $\Delta \boldsymbol{\mu}_{p}$ as follows:

$$
\frac{\partial \mathbf{F}}{\partial \mu_{i}}\left(\hat{\boldsymbol{\mu}}_{p}\right)=\frac{\partial \mathbf{F}}{\partial \mu_{i}}\left(\boldsymbol{\mu}_{p}\right)+\sum_{j=1}^{J} \frac{\partial^{2} \mathbf{F}}{\partial \mu_{i} \partial \mu_{j}}\left(\boldsymbol{\mu}_{p}\right) \Delta \boldsymbol{\mu}_{p}(j)
$$

Combining equations (23) with (25) and using $\hat{\Pi}=\Pi-\Delta \Pi$, we obtain

$$
\operatorname{tr}\left((\boldsymbol{\Pi}-\Delta \boldsymbol{\Pi})\left(\frac{\partial \mathbf{F}}{\partial \mu_{i}}\left(\boldsymbol{\mu}_{p}\right)+\sum_{j=1}^{J} \frac{\partial^{2} \mathbf{F}}{\partial \mu_{i} \partial \mu_{j}}\left(\boldsymbol{\mu}_{p}\right) \Delta \boldsymbol{\mu}_{p}(j)\right)\right)=0 .
$$

By keeping only first order terms, we obtain

$$
\operatorname{tr}\left(\Delta \boldsymbol{\Pi} \frac{\partial \mathbf{F}}{\partial \mu_{i}}\left(\boldsymbol{\mu}_{p}\right)\right)=\sum_{j=1}^{J} \operatorname{tr}\left(\boldsymbol{\Pi} \frac{\partial^{2} \mathbf{F}}{\partial \mu_{i} \partial \mu_{j}}\left(\boldsymbol{\mu}_{p}\right)\right) \Delta \boldsymbol{\mu}_{p}(j)
$$

or equivalently:

$$
\mathrm{g}=\mathrm{B} \Delta \boldsymbol{\mu}_{p}
$$

with $g(i)=\operatorname{tr}\left(\Delta \boldsymbol{\Pi} \frac{\partial \mathbf{F}}{\partial \mu_{i}}\left(\boldsymbol{\mu}_{p}\right)\right)$ and $B(i, j)=\operatorname{tr}\left(\boldsymbol{\Pi} \frac{\partial^{2} \mathbf{F}}{\partial \mu_{i} \partial \mu_{j}}\left(\boldsymbol{\mu}_{p}\right)\right)$, which allows to conclude the proof:

$$
\Delta \boldsymbol{\mu}_{p}=\mathbf{B}^{-1} \mathbf{g}
$$

The previous proposition indicates that the error $\Delta \boldsymbol{\mu}_{p}$ is directly related to the estimation error $\Delta \Pi$ on the projector $\Pi$. The following proposition links $\Delta \Pi$ to the estimation error on the data covariance matrix $\Delta \mathbf{R}=\mathbf{R}-\hat{\mathbf{R}}$.

Proposition 4.2. The covariance matrix of $\Delta \boldsymbol{\mu}_{p}$ is given by:

$$
E\left[\Delta \boldsymbol{\mu}_{p} \Delta \boldsymbol{\mu}_{p}^{H}\right]=\mathbf{B}^{-1} E\left[\mathbf{g g}^{H}\right]\left(\mathbf{B}^{-1}\right)^{H}
$$

with

$$
\begin{aligned}
& E\left[\mathbf{g g}^{H}\right](i, j)=\sum_{k, l, k^{\prime}, l^{\prime}=1}^{L} E\left[\Delta \mathbf{R}(k, l) \Delta \mathbf{R}\left(k^{\prime}, l^{\prime}\right)\right] \\
& .\left(\mathbf{C}_{i}(l, k)+\mathbf{D}_{i}(l, k)\right)\left(\mathbf{C}_{j}\left(l^{\prime}, k^{\prime}\right)+\mathbf{D}_{j}\left(l^{\prime}, k^{\prime}\right)\right)
\end{aligned}
$$

where $\mathbf{C}_{i}=\mathbf{S}^{\dagger} \frac{\partial \mathbf{F}}{\partial \mu_{i}}\left(\boldsymbol{\mu}_{p}\right) \boldsymbol{\Pi} \in \mathbb{C}^{L \times L}, \mathbf{D}_{i}=\boldsymbol{\Pi} \frac{\partial \mathbf{F}}{\partial \mu_{i}}\left(\boldsymbol{\mu}_{p}\right) \mathbf{S}^{\dagger} \in \mathbb{C}^{L \times L}$ and $\mathbf{S}^{\dagger}=$ $\sum_{p=1}^{P} \frac{1}{\sigma_{p}^{2}-\sigma^{2}} \mathbf{u}_{p} \mathbf{u}_{p}^{H} \in \mathbb{C}^{L \times L}$ (with $\left\{\mathbf{u}_{p}\right\}_{1, P}$ are the $P$ first eigenvectors of $\mathbf{R}$ ). 
ProOf. As stated before, the estimation error $\Delta \Pi$ is the result of the estimation error $\Delta \mathbf{R}$. The relation between $\Delta \Pi$ and $\Delta \mathbf{R}$ is given in [24]:

$$
\Delta \Pi=\mathbf{S}^{\dagger} \Delta \mathbf{R} \Pi+\Pi \Delta \mathbf{R} \mathbf{S}^{\dagger}
$$

From Eq. (21) and since the matrix $\mathbf{B}$ is deterministic, we have:

$$
E\left[\Delta \boldsymbol{\mu}_{p} \Delta \boldsymbol{\mu}_{p}^{H}\right]=\mathbf{B}^{-1} E\left[\mathbf{g g}^{H}\right]\left(\mathbf{B}^{-1}\right)^{H}
$$

with

$$
E\left[\operatorname{gg}^{H}\right](i, j)=E\left[\operatorname{tr}\left(\Delta \mathbf{R}\left(\mathbf{C}_{i}+\mathbf{D}_{i}\right)\right) \operatorname{tr}\left(\Delta \mathbf{R}\left(\mathbf{C}_{j}+\mathbf{D}_{j}\right)\right)\right]
$$

Moreover, we have the following relation for all deterministic matrices $\mathbf{B}_{1}, \mathbf{B}_{2} \in$ $\mathbb{C}^{L \times L}$ :

$$
\begin{aligned}
& \left.E\left[\operatorname{tr}\left(\Delta \mathbf{R B}_{1}\right) \operatorname{tr}\left(\Delta \mathbf{R B}_{2}\right)\right)\right]= \\
& \sum_{k, l, k^{\prime}, l^{\prime}=1}^{L} E\left[\Delta \mathbf{R}(k, l) \Delta \mathbf{R}\left(k^{\prime}, l^{\prime}\right)\right] \mathbf{B}_{1}(l, k) \mathbf{B}_{2}\left(l^{\prime}, k^{\prime}\right),
\end{aligned}
$$

which concludes the proof.

This general proposition will be applied to derive the theoretical MSE of both LV-MUSIC and T-MUSIC algorithms. The difference between the performance of these two algorithms comes from the expression of $E\left[\Delta \mathbf{R}(k, l) \Delta \mathbf{R}\left(k^{\prime}, l^{\prime}\right)\right]$ and is explicited hereafter. Also note that, for T-MUSIC, performance differ whether we consider DOA or polarization parameters.

\subsection{Application to LV-MUSIC and T-MUSIC}

\subsubsection{LV-MUSIC}

For LV-MUSIC, we have $\boldsymbol{\mu}=\left[\theta \boldsymbol{\rho}^{T} \boldsymbol{\phi}^{T}\right]^{T}$ and $L=M N_{c}$. The SCM $\hat{\mathbf{R}}$ is Wishart distributed with parameter matrix $\mathbf{R}$ and $N_{s}$ degrees of freedom. The second order moment of its elements is well known to be given by [27]:

$$
E\left[\Delta \mathbf{R}(k, l) \Delta \mathbf{R}\left(k^{\prime}, l^{\prime}\right)\right]=\frac{1}{N_{s}} \mathbf{R}\left(k^{\prime}, l\right) \mathbf{R}\left(k, l^{\prime}\right)
$$

\subsubsection{T-MUSIC}

For the T-MUSIC algorithm, we have shown in lemma 3.1 that the criterion (17) could be decomposed into two criteria (18): the first for DOA's and the second for polarization parameters. 
DoA estimation by T-MUSIC. In this case, $L=M$ and the criterion is:

$$
\hat{H}_{T, D o A}(\theta)=\operatorname{tr}\left(\hat{\boldsymbol{\Pi}}_{1} \mathbf{d}(\theta) \mathbf{d}(\theta)^{H}\right),
$$

where vector $\boldsymbol{\mu}$ is only equal to $\theta$. The derivation of $E\left[\Delta \mathbf{R}(k, l) \Delta \mathbf{R}\left(k^{\prime}, l^{\prime}\right)\right]$ in this case is based on the two following propositions.

Proposition 4.3. Let $\mathbf{R}_{1}=E\left[\mathbf{X}(n) \mathbf{X}(n)^{H}\right] \in \mathbb{C}^{M \times M}$ and $\hat{\mathbf{R}}_{1}$ its estimate obtained from $N_{s}$ i.i.d. samples $\mathbf{X}(n)$, based on the model of $E q$. (14):

$$
\hat{\mathbf{R}}_{1}=\frac{1}{N_{s}} \sum_{n=1}^{N_{s}} \mathbf{X}(n) \mathbf{X}^{H}(n)
$$

The eigenvectors of $[\mathcal{R}]_{1}$ (resp. $[\hat{\mathcal{R}}]_{1}$ ) are equal to those of $\mathbf{R}_{1}$ (resp. $\hat{\mathbf{R}}_{1}$ ). Therefore, the projector $\boldsymbol{\Pi}_{1}$ (resp. $\hat{\Pi}_{1}$ ) can be built from $[\mathcal{R}]_{1}$ (resp. $[\hat{\mathcal{R}}]_{1}$ ) or $\mathbf{R}_{1}$ (resp. $\hat{\mathbf{R}}_{1}$ ).

Proof. $\hat{\boldsymbol{\Pi}}_{1}=\hat{\mathbf{U}}_{0}^{(1)} \hat{\mathbf{U}}_{0}^{(1) H}$ is obtained by the SVD of $[\hat{\mathcal{R}}]_{1} \in \mathbb{C}^{M \times N_{c} M N_{c}}$ which is the unfolding of the SCT $\hat{\mathcal{R}}$ in the first direction:

$$
\begin{aligned}
& {[\hat{\mathcal{R}}]_{1}=\hat{\mathbf{U}}^{(1)} \hat{\boldsymbol{\Sigma}}^{(1)} \hat{\mathbf{V}}^{(1) H}} \\
& \hat{\mathbf{U}}_{0}^{(1)}=\left[\hat{\mathbf{u}}_{P+1}^{(1)}, \ldots, \hat{\mathbf{u}}_{M}^{(1)}\right]
\end{aligned}
$$

Let $\boldsymbol{X} \in \mathbb{C}^{M \times N_{c} \times N_{s}}$ be the tensor composed of all matricial observations $\mathbf{X}(n)$. From [5], $[\hat{\mathcal{R}}]_{1}$ and $[\boldsymbol{X}]_{1}$ share the same left eigenvectors:

$$
\begin{aligned}
& {[\hat{\mathcal{R}}]_{1}=\hat{\mathbf{U}}^{(1)} \hat{\boldsymbol{\Sigma}}^{(1)} \hat{\mathbf{V}}^{(1) H}} \\
& {[\boldsymbol{X}]_{1}=\hat{\mathbf{U}}^{(1)} \hat{\boldsymbol{\Sigma}} \hat{\mathbf{V}}^{H}}
\end{aligned}
$$

Moreover, the left eigenvectors of $[\boldsymbol{X}]_{1}$ are obtained by diagonalizing the matrix $[\boldsymbol{X}]_{1}[\boldsymbol{X}]_{1}^{H}$. But matrix $[\boldsymbol{X}]_{1}[\boldsymbol{X}]_{1}^{H}$ can be rewritten as:

$$
\begin{aligned}
{[\boldsymbol{X}]_{1}[\boldsymbol{X}]_{1}^{H} } & =\sum_{n=1}^{N_{s}} \mathbf{X}(n) \mathbf{X}^{H}(n) \\
& =N_{s} \frac{1}{N_{s}} \sum_{n=1}^{N_{s}} \mathbf{X}(n) \mathbf{X}^{H}(n) . \\
& =N_{s} \hat{\mathbf{R}}_{1}
\end{aligned}
$$

We deduce that $\hat{\mathbf{R}}_{1}$ and $[\hat{\mathcal{R}}]_{1}$ have the same left eigenvectors. The proof for $\mathbf{R}_{1}$ and $[\mathcal{R}]_{1}$ is similar. 
The previous proposition allows to conclude that the values of $E\left[\Delta \mathbf{R}(k, l) \Delta \mathbf{R}\left(k^{\prime}, l^{\prime}\right)\right]$ are equal to $E\left[\Delta \mathbf{R}_{1}(k, l) \Delta \mathbf{R}_{1}\left(k^{\prime}, l^{\prime}\right)\right]$. In the following proposition, we derive therefore the expression of $E\left[\Delta \mathbf{R}_{1}(k, l) \Delta \mathbf{R}_{1}\left(k^{\prime}, l^{\prime}\right)\right]$.

Proposition 4.4. Let $\Delta \mathbf{R}_{1}=\hat{\mathbf{R}}_{1}-\mathbf{R}_{1}$ be the estimation error on $\mathbf{R}_{1}$. The second order moments of $\Delta \mathbf{R}_{1}$ are given by:

$$
\begin{aligned}
& N_{s} E\left(\Delta \mathbf{R}_{1}(k, l) \Delta \mathbf{R}_{1}\left(k^{\prime}, l^{\prime}\right)\right)= \\
& \sum_{p, p^{\prime}=1}^{P} \sigma_{p}^{2} \sigma_{p^{\prime}}^{2}\left(\mathbf{A}_{p} \mathbf{A}_{p^{\prime}}^{H}\right)(k, l)\left(\mathbf{A}_{p^{\prime}} \mathbf{A}_{p}^{H}\right)\left(k^{\prime}, l^{\prime}\right) \\
& +\sum_{p=1}^{P} \sigma_{p}^{2}\left(\mathbf{A}_{p} \mathbf{A}_{p}^{H}\right)\left(k, l^{\prime}\right) \sigma^{2} \delta\left(k^{\prime}, l\right) \\
& +\sum_{p=1}^{P} \sigma_{p}^{2}\left(\mathbf{A}_{p} \mathbf{A}_{p}^{H}\right)\left(k^{\prime}, l\right) \sigma^{2} \delta\left(k, l^{\prime}\right) \\
& +N_{c} \sigma^{4} \delta\left(k^{\prime}, l\right) \delta\left(k, l^{\prime}\right), \quad \forall k, l, k^{\prime}, l^{\prime}=1 \ldots M .
\end{aligned}
$$

where $\mathbf{A}_{p}=\mathbf{A}\left(\theta_{p}, \boldsymbol{\rho}(p), \boldsymbol{\phi}(p)\right)$.

PROOF. The proof is given in the appendix.

Polarization parameters estimation by T-MUSIC. In this case, $L=N_{c}$ and the criterion is:

$$
H_{T, P o l a r}(\boldsymbol{\rho}, \boldsymbol{\phi})=\operatorname{tr}\left(\hat{\boldsymbol{\Pi}}_{2} \mathbf{p}(\boldsymbol{\rho}, \boldsymbol{\phi}) \mathbf{p}(\boldsymbol{\rho}, \boldsymbol{\phi})^{H}\right)
$$

where vector $\boldsymbol{\mu}$ is equal to $\left[\boldsymbol{\rho}^{T} \boldsymbol{\phi}^{T}\right]^{T}$. The derivation of $E\left[\Delta \mathbf{R}(k, l) \Delta \mathbf{R}\left(k^{\prime}, l^{\prime}\right)\right]$ in this case is based on the two following propositions.

Proposition 4.5. Let $\mathbf{R}_{2}=E\left[\mathbf{X}^{T}(n) \mathbf{X}^{*}(n)\right] \in \mathbb{C}^{N_{c} \times N_{c}}$ and $\hat{\mathbf{R}}_{2}$ its estimate obtained from $N_{s}$ i.i.d. samples $\mathbf{X}(n)$, based on the model of $E q$. (14):

$$
\hat{\mathbf{R}}_{2}=\frac{1}{N_{s}} \sum_{n=1}^{N_{s}} \mathbf{X}^{T}(n) \mathbf{X}^{*}(n)
$$

The eigenvectors of $[\mathcal{R}]_{2}$ (resp. $[\hat{\mathcal{R}}]_{2}$ ) are equal to those of $\mathbf{R}_{2}$ (resp. $\left.\hat{\mathbf{R}}_{2}\right)$. Therefore, the projector $\boldsymbol{\Pi}_{2}$ (resp. $\hat{\Pi}_{2}$ ) can be built from $[\mathcal{R}]_{2}$ (resp. $[\hat{\mathcal{R}}]_{2}$ ) or $\mathbf{R}_{2}$ (resp. $\hat{\mathbf{R}}_{2}$ ).

PROOF. The proof is similar to that of proposition 4.3 and is omitted. 
In the following proposition, we express $E\left[\Delta \mathbf{R}_{2}(k, l) \Delta \mathbf{R}_{2}\left(k^{\prime}, l^{\prime}\right)\right]$.

Proposition 4.6. Let $\Delta \mathbf{R}_{2}=\hat{\mathbf{R}}_{2}-\mathbf{R}_{2}$ be the estimation error on $\mathbf{R}_{2}$. The second order moments of $\Delta \mathbf{R}_{2}$ are given by:

$$
\begin{aligned}
& N_{s} E\left(\Delta \mathbf{R}_{2}(k, l) \Delta \mathbf{R}_{2}\left(k^{\prime}, l^{\prime}\right)\right)= \\
& \sum_{p, p^{\prime}=1}^{P} \sigma_{p}^{2} \sigma_{p^{\prime}}^{2}\left(\mathbf{A}_{p}^{T} \mathbf{A}_{p^{\prime}}^{*}\right)(k, l)\left(\mathbf{A}_{p^{\prime}}^{T} \mathbf{A}_{p}^{*}\right)\left(k^{\prime}, l^{\prime}\right) \\
& +\sum_{p=1}^{P} \sigma_{p}^{2}\left(\mathbf{A}_{p}^{T} \mathbf{A}_{p}^{*}\right)\left(k, l^{\prime}\right) \sigma^{2} \delta\left(l, k^{\prime}\right) \\
& +\sum_{p=1}^{P} \sigma_{p}^{2}\left(\mathbf{A}_{p}^{T} \mathbf{A}_{p}^{*}\right)\left(k^{\prime}, l\right) \sigma^{2} \delta\left(l^{\prime}, k\right) \\
& +M \sigma^{4} \delta\left(k^{\prime}, l\right) \delta\left(k, l^{\prime}\right) .
\end{aligned}
$$

where $\mathbf{A}_{p}=\mathbf{A}\left(\theta_{p}, \boldsymbol{\rho}(p), \boldsymbol{\phi}(p)\right)$.

PROOF. The proof is similar to that of proposition 4.4 and is omitted.

\section{Simulations}

We consider an Uniform Linear Array (ULA) composed of $M=10$ sensors, which are able to receive in $\mathrm{H}$ and $\mathrm{V}$ polarizations: this results in $N_{c}=3$. The wavelength is equal to $\lambda=0.1 \mathrm{~m}$. The spacing between inter-sensor is $d=\frac{\lambda}{2}$. The number of secondary data is $N_{s}=300$. When we consider more than 1 source, we assume that they will have the same power. The Signal to Noise Ratio is defined by:

$$
S N R=\frac{\sigma_{p}^{2}}{\sigma^{2}}
$$

First, we validate the presented theoretical performance of LV-MUSIC and T-MUSIC. This study focuses on the the DOA and the polarization parameters estimation. We consider $P=1$ source with a DoA of $\theta=-3^{\circ}$. The polarization parameters are $\boldsymbol{\rho}=\left[\begin{array}{lll}1 & 1 & 1\end{array}\right]^{T}$ and $\boldsymbol{\phi}=\left[\begin{array}{l}0-0.2 \mathrm{rad} 0.2 \mathrm{rad}\end{array}\right]^{T}$. Propositions 4.2, 4.4 and 4.6 are used to compute the theoretical MSEs of the DoA and the polarization parameters for LV-MUSIC and T-MUSIC. The experimental MSEs of LV-MUSIC and T-MUSIC are computed from $N_{\text {rea }}=1000$ Monte-Carlo trials by minimizing criteria (13) and (18). We define the MSE for $\theta$ as:

$$
M S E_{\theta}=E\left[|\hat{\theta}-\theta|^{2}\right]
$$


and for the polarization parameters $\rho$ and $\phi$ as:

$$
\begin{aligned}
M S E_{\rho} & =\frac{1}{2}\left(E\left[|\hat{\rho}(2)-\rho(2)|^{2}\right]+E\left[|\hat{\rho}(3)-\rho(3)|^{2}\right]\right) \\
M S E_{\phi} & =\frac{1}{2}\left(E\left[|\hat{\phi}(2)-\phi(2)|^{2}\right]+E\left[|\hat{\phi}(3)-\phi(3)|^{2}\right]\right)
\end{aligned}
$$

Figure 1 shows the experimental and theoretical MSEs of the DOA for LVMUSIC and T-MUSIC. This figure illustrates the validity of the presented results since at high SNR the experimental and theoretical MSEs are identical. We notice that performance of LV-MUSIC and T-MUSIC are very close for 1 source. Figure 2 shows the mean of the experimental and theoretical MSEs of the 4 polarization parameters $\rho(2), \rho(3), \phi(2), \phi(3)$ for LV-MUSIC and T-MUSIC. As for DOAs, this simulation validates our theoretical result for the polarization parameters estimated by T-MUSIC and LV-MUSIC.

Now, we investigate the performance of both algorithms when two sources are present. First, we consider two sources having the same polarization parameters. Figures 3 and 4 show the theoretical MSEs of all parameters for LV-MUSIC and TMUSIC as a function of the sources separation in degree. For these configurations and parameters, one may notice that T-MUSIC outperforms the LV-MUSIC in terms of estimation accuracy, which illustrates its practical interest.

Figures 5 and 6 still show the theoretical MSEs of all parameters for LVMUSIC and T-MUSIC as a function of the sources separation. The two sources now have different polarization parameters. We conclude that LV-MUSIC achieves better performance than T-MUSIC for DoA estimation but we have the opposite conclusion for the polarization parameters estimation.

Let us comment the obtained results. One problem with $L V-M U S I C$ is that the data size has been increased $\left(M N_{c}\right.$ instead of $M$ for a classical DOA problem). Therefore, the estimation of the covariance matrix (and that of $\hat{\Pi}$ ) requires more samples than for classical MUSIC. The interest of T-MUSIC is that the data size reduces to $M$ for DOA estimation and to $N_{c}$ for polarization parameters estimation. Therefore, less samples are needed for a good estimation of the subspace projectors $\hat{\Pi}_{1}$ and $\hat{\Pi}_{2}$ : this is a positive point for T-MUSIC. On the other hand, LV-MUSIC takes full advantage of the possibility to resolve sources jointly in the angular and polarization domains through the composite steering vector of Eq. (8): closely spaced sources may have almost orthogonal steering vectors (8) when their polarization parameters are different enough. This is not the case for T-MUSIC which treats separately angles and polarizations, and it turns out to be a drawback compared to LV-MUSIC. So, depending on the scenario, T-MUSIC or LV-MUSIC may exhibit superior theoretical performance. 


\section{Conclusion}

In this paper, we have derived the theoretical performance of a Tensor MUSIC algorithm based on the Higher Order Singular Value Decomposition (HOSVD). This derivation has been performed by means of a perturbation analysis and has lead to the theoretical MSEs for DOAs and polarization parameters of polarized sources. These results allow a comparison of the theoretical performance of TMUSIC and LV-MUSIC algorithms in a given scenario. Numerical simulations have illustrated the agreement between theoretical results and experimental MSEs.

\section{appendix: proof of proposition 4.4}

Let $\mathbf{X}(n)$ be the random matrix defined by (14). From (38) we have:

$$
E\left[\hat{\mathbf{R}}_{1}\right]=E\left[\mathbf{X}(n) \mathbf{X}^{H}(n)\right]=\mathbf{R}_{1}
$$

and consequently

$$
E\left(\Delta \mathbf{R}_{1}\right)=0
$$

Let us now investigate the second order moments of $\Delta \mathbf{R}_{1}$. Let us set $\mathrm{X}$ a random matrix sharing the same distribution as $\mathbf{X}(n)$. We have obviously:

$$
\begin{aligned}
& E\left[\Delta \mathbf{R}_{1}(k, l) \Delta \mathbf{R}_{1}\left(k^{\prime}, l^{\prime}\right)\right]= \\
& \frac{1}{N_{s}}\left(E\left[\left(\mathbf{X X}^{H}\right)(k, l)\left(\mathbf{X X}^{H}\right)\left(k^{\prime}, l^{\prime}\right)\right]-\mathbf{R}_{1}(k, l) \mathbf{R}_{1}\left(k^{\prime}, l^{\prime}\right)\right)
\end{aligned}
$$

where $\mathbf{R}_{1}$ is easily obtained from (14):

$$
\mathbf{R}_{1}=E\left[\mathbf{X X}^{H}\right]=\sum_{p=1}^{P} \sigma_{p}^{2} \mathbf{A}_{p} \mathbf{A}_{p}^{H}+N_{c} \sigma^{2} \mathbf{I}_{M},
$$

where $\mathbf{A}_{p}=\mathbf{A}\left(\theta_{p}, \boldsymbol{\rho}_{p}, \boldsymbol{\phi}_{p}\right)$. This leads, in Eq. (53), to:

$$
\begin{aligned}
\mathbf{R}_{1}(k, l) \mathbf{R}_{1}\left(k^{\prime}, l^{\prime}\right)= & \sum_{p, p^{\prime}=1}^{P} \sigma_{p}^{2} \sigma_{p^{\prime}}^{2}\left(\mathbf{A}_{p} \mathbf{A}_{p}^{H}\right)(k, l)\left(\mathbf{A}_{p^{\prime}} \mathbf{A}_{p^{\prime}}^{H}\right)\left(k^{\prime}, l^{\prime}\right) \\
& +\sum_{p=1}^{P} \sigma_{p}^{2}\left(\mathbf{A}_{p} \mathbf{A}_{p}^{H}\right)(k, l) N_{c} \sigma^{2} \delta\left(k^{\prime}, l^{\prime}\right) \\
& +\sum_{p=1}^{P} \sigma_{p}^{2}\left(\mathbf{A}_{p} \mathbf{A}_{p}^{H}\right)\left(k^{\prime}, l^{\prime}\right) N_{c} \sigma^{2} \delta(k, l) \\
& +\sigma^{4} N_{c}^{2} \delta(k, l) \delta\left(k^{\prime}, l^{\prime}\right)
\end{aligned}
$$


Table 1: Vanishing terms in $E\left[\left(\mathbf{X X}^{H}\right)(k, l)\left(\mathbf{X X}^{H}\right)\left(k^{\prime}, l^{\prime}\right)\right]$ : 0 indicates terms with a vanishing expectation.

\begin{tabular}{|c|c|c|c|c|}
\hline$E[\ldots]$ & $(56)^{\prime}$ & $(57)^{\prime}$ & $(58)^{\prime}$ & $(59)^{\prime}$ \\
\hline$(56)$ & $\times$ & 0 & 0 & $\times$ \\
\hline$(57)$ & 0 & 0 & $\times$ & 0 \\
\hline$(58)$ & 0 & $\times$ & 0 & 0 \\
\hline$(59)$ & $\times$ & 0 & 0 & $\times$ \\
\hline
\end{tabular}

Let us turn to the term $E\left[\left(\mathbf{X X}^{H}\right)(k, l)\left(\mathbf{X X}^{H}\right)\left(k^{\prime}, l^{\prime}\right)\right]$ in (53). First, $\left(\mathbf{X X}^{H}\right)(k, l)$ can be decomposed as the sum of 4 terms:

$$
\begin{array}{r}
\left(\mathbf{X X}^{H}\right)(k, l)=\sum_{p, p^{\prime}=1}^{P} s_{p^{\prime}} s_{p^{\prime}}^{*}\left(\mathbf{A}_{p} \mathbf{A}_{p^{\prime}}^{H}\right)(k, l)+ \\
\sum_{p=1}^{P} s_{p}\left(\mathbf{A}_{p} \mathbf{N}^{H}\right)(k, l)+ \\
\sum_{p=1}^{P} s_{p}^{*}\left(\mathbf{N A}_{p}^{H}\right)(k, l)+ \\
\left(\mathbf{N N}^{H}\right)(k, l) .
\end{array}
$$

In the same way, the corresponding terms of $\left(\mathbf{X X}^{H}\right)\left(k^{\prime}, l^{\prime}\right)$ are numbered (56)', (57)', (58)' and (59)'. The derivation of $E\left[\left(\mathbf{X X}^{H}\right)(k, l)\left(\mathbf{X X}^{H}\right)\left(k^{\prime}, l^{\prime}\right)\right]$ is the sum of 16 terms $E\left[(56)(56)^{\prime}\right], E\left[(56)(57)^{\prime}\right], \ldots, E\left[(59)(59)^{\prime}\right]$ out of which only 6 of them are non-zero: they are listed in Table 1.

Therefore $E\left[\left(\mathbf{X X}^{H}\right)(k, l)\left(\mathbf{X}(k) \mathbf{X}^{H}(k)\right)\left(k^{\prime}, l^{\prime}\right)\right]$ reduces to:

$$
\begin{aligned}
E\left[\left(\mathbf{X X}^{H}\right)(k, l)\left(\mathbf{X}(k) \mathbf{X}^{H}(k)\right)\left(k^{\prime}, l^{\prime}\right)\right] & \\
E\left[(56)(56)^{\prime}\right]+ & E\left[(56)(59)^{\prime}\right] \\
+E\left[(56)^{\prime}(59)\right]+ & E\left[(57)(58)^{\prime}\right] \\
& +E\left[(57)^{\prime}(58)\right]+E\left[(59)(59)^{\prime}\right] .
\end{aligned}
$$

It remains 4 terms to compute since the two last one could be deducing from the 
others by inverting $k, l$ and $k^{\prime}, l^{\prime}$.

$$
\begin{gathered}
E\left[(56)(56)^{\prime}\right]= \\
=\sum_{p, p^{\prime}=1}^{P} \sigma_{p}^{2} \sigma_{p^{\prime}}^{2}\left(\mathbf{A}_{p} \mathbf{A}_{p}^{H}\right)(k, l)\left(\mathbf{A}_{p^{\prime}} \mathbf{A}_{p^{\prime}}^{H}\right)\left(k^{\prime}, l^{\prime}\right) \\
+\sum_{p, p^{\prime}=1}^{P} \sigma_{p}^{2} \sigma_{p^{\prime}}^{2}\left(\mathbf{A}_{p} \mathbf{A}_{p^{\prime}}^{H}\right)(k, l)\left(\mathbf{A}_{p^{\prime}} \mathbf{A}_{p}^{H}\right)\left(k^{\prime}, l^{\prime}\right) \\
E\left[(56)(59)^{\prime}\right]=\sum_{p=1}^{P} \sigma_{p}^{2}\left(\mathbf{A}_{p} \mathbf{A}_{p}^{H}\right)(k, l) N_{c} \sigma^{2} \delta\left(k^{\prime}, l^{\prime}\right) \\
E\left[(57)(58)^{\prime}\right]=\sum_{p=1}^{P} \sigma_{p}^{2}\left(\mathbf{A}_{p} \mathbf{A}_{p}^{H}\right)\left(k, l^{\prime}\right) \sigma^{2} \delta\left(k^{\prime}, l\right) \\
E\left[(59)(59)^{\prime}\right]=N_{c} \sigma^{4} \delta\left(k, l^{\prime}\right) \delta\left(k^{\prime}, l\right)+N_{c}^{2} \sigma^{4} \delta(k, l) \delta\left(k^{\prime}, l^{\prime}\right) .
\end{gathered}
$$

We finally obtain:

$$
\begin{aligned}
E\left[\left(\mathbf{X X}^{H}\right)(k, l)\left(\mathbf{X X}^{H}\right)\left(k^{\prime}, l^{\prime}\right)\right]= & \sum_{p, p^{\prime}=1}^{P} \sigma_{p}^{2} \sigma_{p^{\prime}}^{2}\left(\mathbf{A}_{p} \mathbf{A}_{p}^{H}\right)(k, l)\left(\mathbf{A}_{p^{\prime}} \mathbf{A}_{p^{\prime}}^{H}\right)\left(k^{\prime}, l^{\prime}\right) \\
& \sum_{p, p^{\prime}=1}^{P} \sigma_{p}^{2} \sigma_{p^{\prime}}^{2}\left(\mathbf{A}_{p} \mathbf{A}_{p^{\prime}}^{H}\right)(k, l)\left(\mathbf{A}_{p^{\prime}} \mathbf{A}_{p}^{H}\right)\left(k^{\prime}, l^{\prime}\right) \\
& +\sum_{p=1}^{P} \sigma_{p}^{2}\left(\mathbf{A}_{p} \mathbf{A}_{p}^{H}\right)(k, l) N_{c} \sigma^{2} \delta\left(k^{\prime}, l^{\prime}\right) \\
& +\sum_{p=1}^{P} \sigma_{p}^{2}\left(\mathbf{A}_{p} \mathbf{A}_{p}^{H}\right)\left(k^{\prime}, l^{\prime}\right) N_{c} \sigma^{2} \delta(k, l) \\
& +\sum_{p=1}^{P} \sigma_{p}^{2}\left(\mathbf{A}_{p} \mathbf{A}_{p}^{H}\right)\left(k, l^{\prime}\right) \sigma^{2} \delta\left(k^{\prime}, l\right) \\
& +\sum_{p=1}^{P} \sigma_{p}^{2}\left(\mathbf{A}_{p} \mathbf{A}_{p}^{H}\right)\left(k^{\prime}, l\right) \sigma^{2} \delta\left(k, l^{\prime}\right) \\
& +N_{c} \sigma^{4} \delta\left(k^{\prime}, l\right) \delta\left(k, l^{\prime}\right)+N_{c}^{2} \sigma^{4} \delta(k, l) \delta\left(k^{\prime}, l^{\prime}\right) .
\end{aligned}
$$


The final result follows from Eqs. (55), (64) and (53):

$$
\begin{array}{r}
N_{s} E\left(\Delta \mathbf{R}_{1}(k, l) \Delta \mathbf{R}_{1}\left(k^{\prime}, l^{\prime}\right)\right)= \\
\sum_{p, p^{\prime}=1}^{P} \sigma_{p}^{2} \sigma_{p^{\prime}}^{2}\left(\mathbf{A}_{p} \mathbf{A}_{p^{\prime}}^{H}\right)(k, l)\left(\mathbf{A}_{p^{\prime}} \mathbf{A}_{p}^{H}\right)\left(k^{\prime}, l^{\prime}\right) \\
+\sum_{p=1}^{P} \sigma_{p}^{2}\left(\mathbf{A}_{p} \mathbf{A}_{p}^{H}\right)\left(k, l^{\prime}\right) \sigma^{2} \delta\left(k^{\prime}, l\right) \\
+\sum_{p=1}^{P} \sigma_{p}^{2}\left(\mathbf{A}_{p} \mathbf{A}_{p}^{H}\right)\left(k^{\prime}, l\right) \sigma^{2} \delta\left(k, l^{\prime}\right) \\
+N_{c} \sigma^{4} \delta\left(k^{\prime}, l\right) \delta\left(k, l^{\prime}\right) .
\end{array}
$$

\section{References}

[1] N. Sidiropoulos, R. Bro, G. Giannakis, Parallel factor analysis in sensor array processing, IEEE Trans. Signal Process. 48 (8) (2000) 2377-2388.

[2] N. Sidiropoulos, R. Bro, G. Giannakis, Blind PARAFAC receivers for DSCDMA systems, IEEE Trans. Signal Process. 48 (3) (2000) 810-823.

[3] G. Showman, W. Melvin, M. Belenkii, Performance evaluation of two polarimetric STAP architectures, in: Proc. of the IEEE Int. Radar Conf., 2003, pp. 59-65.

[4] S. Miron, N. L. Bihan, J. I. Mars, Vector-sensor MUSIC for polarized seismic sources localisation, EURASIP Journal of Applied Signal Processing (1) (2005) $74-84$.

[5] M. Haardt, F. Roemer, G. D. Galdo, Higher-order SVD-based subspace estimation to improve the parameter estimation accuracy in multidimensionnal harmonic retrieval problems, IEEE Trans. Signal Process. 56 (7) (2008) 3198-3213.

[6] R. Boyer, Decoupled root-music algorithm for multidimensional harmonic retrieval, in: IEEE 9th Workshop on Signal Process. Advances in Wireless Communications (SPAWC), 2008. Recife, Brazil., 2008, pp. 16-20.

[7] G. Favier, M. D. Costa, A. D. Almeida, J. Romano, Tensor space time (TST) coding for MIMO wireless communication systems, Signal Processing 92 (4) (2012) 1079 - 1092. 
[8] L. D. Lathauwer, B. D. Moor, J. Vandewalle, A multilinear singular value decomposition, SIAM J. Matrix Anal. Apl. 21 (4) (2000) 1253-1278.

[9] M. Papy, L. D. Lathauwer, S. V. Huffel, Exponential data fitting using multilinear algebra: The single-channel and multi-channel case, Numer. Linear Algebra Appl. 12 (2005) 809-826.

[10] T. Kolfa, B. Bader, Tensor decompositions and applications, SIAM Review 51 (2009) $455-500$.

[11] P. Comon, Tensors: a brief introduction, IEEE Signal Processing Magazine 31 (2) (2014) 44-53.

[12] R. Harshman, Foundations of the PARAFAC procedure: Models and conditions for an 'explanatory' multi-modal analysis, UCLA Working Papers in Phonetics 16 (1970) 1-84.

[13] L. Tucker, Some mathematical notes on three-mode factor analysis, Psychometrika 31 (1966) 279-311.

[14] R. Schmidt, Multiple emitter location and signal parameter estimation, IEEE Trans.-ASSP 34 (3) (1986) 276 - 280.

[15] I. Reed, J. Mallett, L. Brennan, Rapid convergence rate in adaptive arrays, IEEE Trans. on Aero. and Elec. Syst. AES-10 (6) (1974) 853 - 863.

[16] S. Miron, X. Guo, D. Brie, DOA estimation for polarized sources on a vector-sensor array by PARAFAC decomposition of the fourth-order covariance tensor, in: Proceedings of EUSIPCO, Lausanne, Switzerland, 2008.

[17] X. Gong, Z. Liu, Y. Xu, M. I.Ahmad, Direction-of-arrival estimation via twofold mode-projection, Signal Processing 89 (5) (2009) 931-842.

[18] M. Boizard, G. Ginolhac, F. Pascal, S. Miron, P. Forster, Numerical performance of a tensor MUSIC algorithm based on HOSVD for a mixture of polarized sources, in: EUSIPCO 2013, Marrakech, Morocco, 2013.

[19] L. D. Lathauwer, B. D. Moor, J. Vandewalle, On the best rank-1 and rank- $\left(r_{1}, r_{2}, \ldots, r_{n}\right)$ approximation and applications of higher-order tensors, SIAM J. Matrix Anal. Apl. 21 (4) (2000) 1324-1342. 
[20] K. Han, A. Nehorai, Nested vector-sensor array processing via tensor modeling, IEEE Trans. Signal Process. 62 (10) (2014) 2542-2553.

[21] F. Li, H. Liu, R. J. Vaccaro, Performance analysis for DOA estimation algorithms: Unification, simplifications, observations, IEEE Trans. on Aero. and Elec. Syst. 29 (4) (1993) 1170 - 1184.

[22] W. Sun, H. So, F. Chan, L. Huang, Tensor approach for eigenvector-based multi-dimensional harmonic retrieval, IEEE Trans. Signal Process. 61 (13) (2013) 3378-3388.

[23] F. Roemer, M. Haardt, G. D. Galdo, Analytical performance assessment of multi-dimensional matrix- and tensor-based ESPRIT-type algorithms, IEEE Trans. Signal Process. 62 (10) (2014) 2611 - 2625.

[24] H. Krim, P. Forster, J. Proakis, Operator approach to performance analysis of root-MUSIC and root-min-norm, IEEE Trans. Signal Process. 40 (7) (1992) $1687-1696$.

[25] G. Ginolhac, P. Forster, F. Pascal, J. Ovarlez, Performance of two low-rank STAP filters in a heterogeneous noise, IEEE Trans. Signal Process. 61 (1) (2013) $57-61$.

[26] G. Ginolhac, P. Forster, F. Pascal, J. Ovarlez, Exploiting persymmetry for low-rank space time adaptive processing, Signal Processing 97 (4) (2014) $242-251$.

[27] D. Maiwald, D. Kraus, On moments of complex Wishart and complex inverse Wishart distributed matrices, in: Acoustics, Speech and Signal Processing, 1977. ICASSP 1977. IEEE International Conference on, 1997, pp. $3817-3820$. 


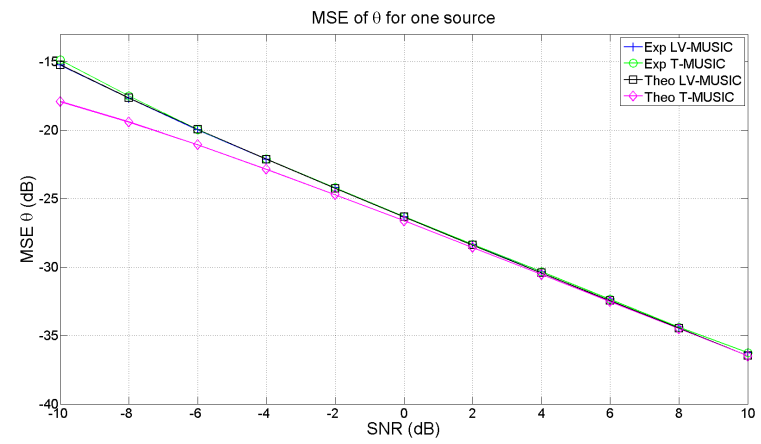

Figure 1: Theoretical and experimental MSEs of DoA as a function of the SNR. The DoA is equal to $\theta=-3^{\circ}$. Its polarization parameters are $\boldsymbol{\rho}=(1,1,1), \boldsymbol{\varphi}=$ $(0,-0.2 \mathrm{rad}, 0.2 \mathrm{rad})$. The other parameters of the simulation are: $N_{s}=300$, $N_{\text {rea }}=10000$. 


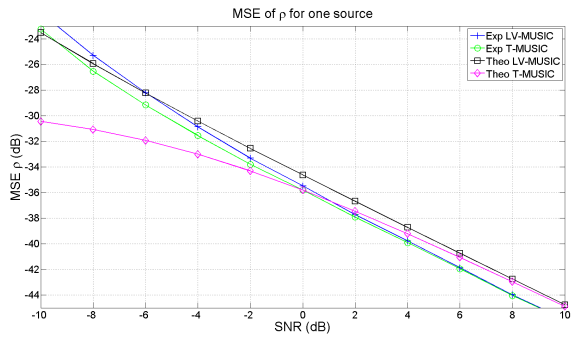

(a)

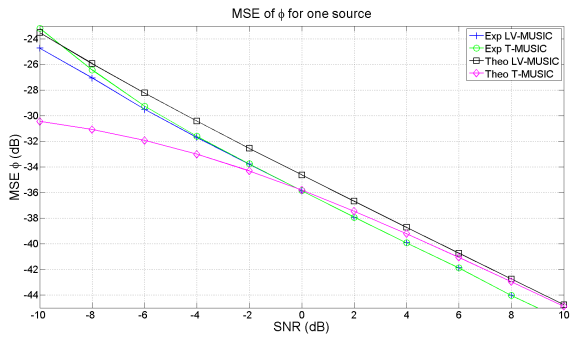

(b)

Figure 2: Theoretical MSEs of polarization parameters ( $\rho$ in (a) and $\phi$ in (b)) as a function of the SNR. The DoA is equal to $\theta=-3^{\circ}$. Its polarization parameters are $\boldsymbol{\rho}=(1,1,1), \boldsymbol{\varphi}=(0,-0.2 \mathrm{rad}, 0.2 \mathrm{rad})$. The other parameters of the simulation are: $N_{s}=300, N_{\text {rea }}=10000$. 


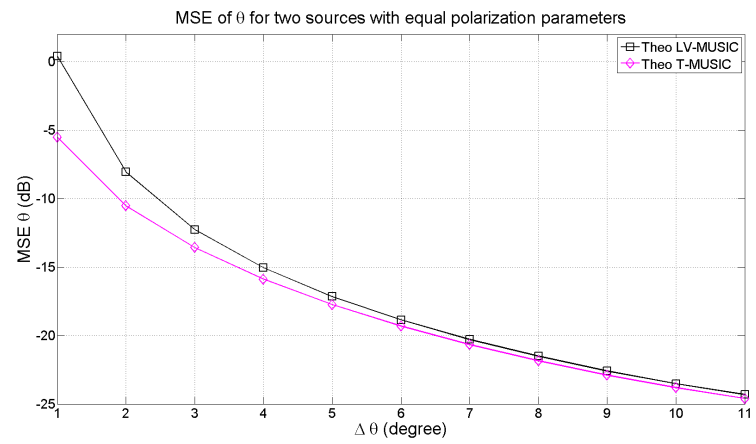

Figure 3: Theoretical MSEs of DoAs as a function of the sources separation. The SNR is equal to $0 \mathrm{~dB}$. The polarization parameters of the two sources are the same and are equal to $\boldsymbol{\rho}_{1}=\boldsymbol{\rho}_{2}=(1,1,1), \boldsymbol{\varphi}_{1}=\boldsymbol{\varphi}_{2}=(0,-0.2 \mathrm{rad}, 0.2 \mathrm{rad})$. The number of secondary data is $N_{s}=300$. 


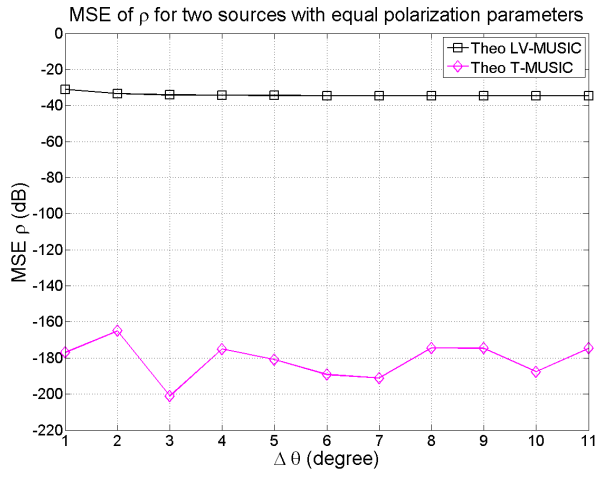

(a)

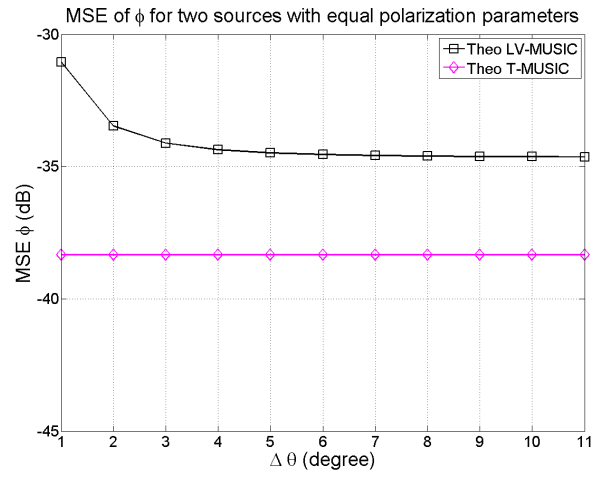

(b)

Figure 4: Theoretical MSEs of polarization parameters ( $\rho$ in (a) and $\phi$ in (b)) as a function of the sources separation. The SNR is equal to $0 \mathrm{~dB}$. The polarization parameters of the two sources are the same and are equal to $\boldsymbol{\rho}_{1}=\boldsymbol{\rho}_{2}=(1,1,1)$, $\varphi_{1}=\varphi_{2}=(0,-0.2 \mathrm{rad}, 0.2 \mathrm{rad})$. The number of secondary data is $N_{s}=300$. 


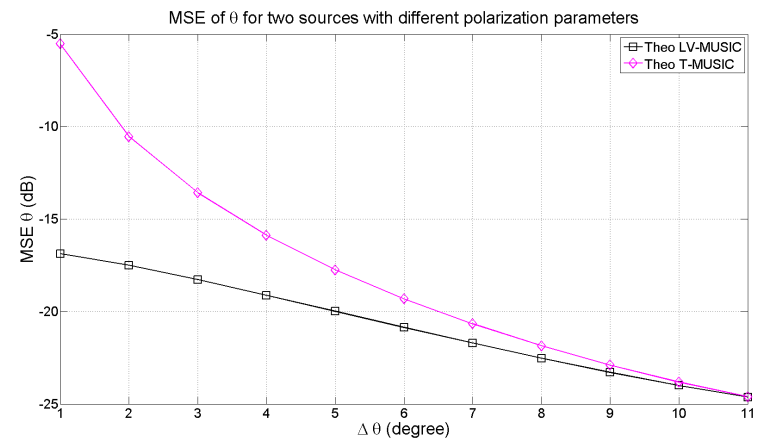

Figure 5: Theoretical MSEs of DoA as a function of the sources separation. The SNR is equal to $0 \mathrm{~dB}$. The polarization parameters of the two sources are equal to $\boldsymbol{\rho}_{1}=(1,1,1), \boldsymbol{\varphi}_{1}=(0,-0.2 \mathrm{rad}, 0.25 \mathrm{rad})$ for source 1 and $\boldsymbol{\rho}_{2}=(1,1,1)$, $\boldsymbol{\varphi}_{2}=(0,0.2 \mathrm{rad},-0.25 \mathrm{rad})$ for source 2 . The number of secondary data is $N_{s}=300$. 


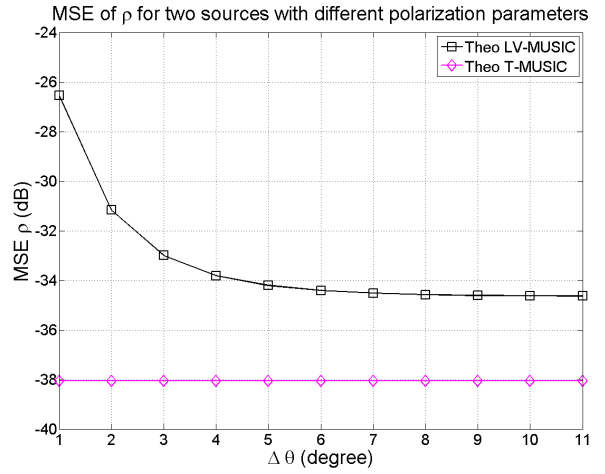

(a)

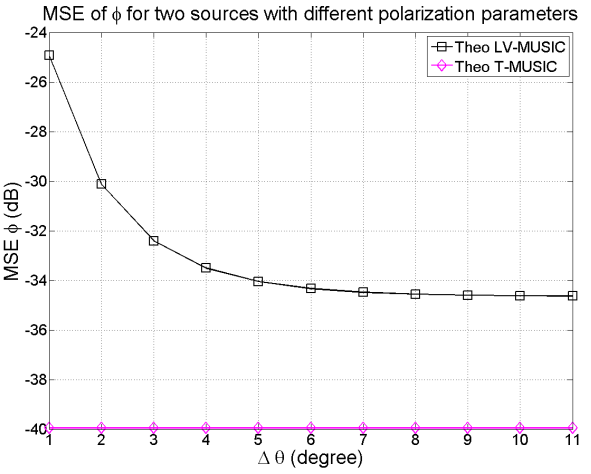

(b)

Figure 6: Theoretical MSEs of polarization parameters ( $\rho$ in (a) and $\phi$ in (b)) as a function of the sources separation. The SNR is equal to $0 \mathrm{~dB}$. The polarization parameters of the two sources are equal to $\boldsymbol{\rho}_{1}=(1,1,1)$, $\boldsymbol{\varphi}_{1}=(0,-0.2 \mathrm{rad}, 0.25 \mathrm{rad})$ for source 1 and $\boldsymbol{\rho}_{2}=(1,1,1), \boldsymbol{\varphi}_{2}=$ $(0,0.2 \mathrm{rad},-0.25 \mathrm{rad})$ for source 2 . The number of secondary data is $N_{s}=300$. 